\title{
DRHotNet: An R package for detecting differential risk hotspots on a linear network
}

\author{
by Álvaro Briz-Redón, Francisco Martínez-Ruiz and Francisco Montes
}

\begin{abstract}
One of the most common applications of spatial data analysis is detecting zones, at a certain scale, where a point-referenced event under study is especially concentrated. The detection of such zones, which are usually referred to as hotspots, is essential in certain fields such as criminology, epidemiology, or traffic safety. Traditionally, hotspot detection procedures have been developed over areal units of analysis. Although working at this spatial scale can be suitable enough for many research or practical purposes, detecting hotspots at a more accurate level (for instance, at the road segment level) may be more convenient sometimes. Furthermore, it is typical that hotspot detection procedures are entirely focused on the determination of zones where an event is (overall) highly concentrated. It is less common, by far, that such procedures focus on detecting zones where a specific type of event is overrepresented in comparison with the other types observed, which have been denoted as differential risk hotspots. The R package DRHotNet provides several functionalities to facilitate the detection of differential risk hotspots within a linear network. In this paper, DRHotNet is depicted, and its usage in the $\mathrm{R}$ console is shown through a detailed analysis of a crime dataset.
\end{abstract}

\section{Introduction}

Hotspot detection consists of finding zones across space where a certain event is highly concentrated. There exists a wide variety of methods in the literature that allow researchers to identify hotspots at a certain level of accuracy or spatial aggregation. Some of them have been massively used in the last decades, including certain local indicators of spatial association such as LISA (Anselin, 1995) or the Getis-Ord statistic (Getis and Ord, 1992), and the spatial scan statistic (Kulldorff, 1997). The first two of these methods are implemented in the R package spdep (Bivand et al., 2013), whereas the scan statistic is implemented in DCluster (Gómez-Rubio et al., 2005) (although other R packages also provide an implementation of these methods). Furthermore, many new $\mathrm{R}$ packages focused on hotspot detection have been released in the last few years. Most of them are model-based and oriented to disease mapping studies that are carried out over administrative (areal) units (Allévius, 2018; Gómez-Rubio et al., 2019; Meyer et al., 2017).

However, the analysis of certain types of events requires detecting hotspots at a level of spatial accuracy greater than that provided by administrative or regular areal units. Indeed, many research studies of the fields of criminology (Andresen et al., 2017; Weisburd, 2015) and traffic safety (BrizRedón et al., 2019b; Nie et al., 2015; Xie and Yan, 2013) that have been published in recent years were entirely carried out on road network structures rather than on administrative units. More specifically, some quantitative criminologists have estimated that around $60 \%$ of the total variability in crime incidence occurs at the street segment level (Schnell et al., 2017; Steenbeek and Weisburd, 2016). A fact that shows the essentiality of using road segments instead of areal structures to properly capture the spatial concentration of certain events.

Fortunately, road network structures were introduced in the context of spatial statistics some years ago, providing the basis for analyzing events lying on such structures, which are usually referred to as linear networks. Indeed, a planar linear network, $L$, is defined as a finite collection of line segments, $L=\cup_{i=1}^{n} l_{i}$, in which each segment contains the points $l_{i}=\left[u_{i}, v_{i}\right]=\left\{t u_{i}+(1-t) v_{i}: t \in[0,1]\right\}$ (Ang et al., 2012; Baddeley et al., 2015, 2017). Following graph theory nomenclature, these segments are sometimes referred to as the edges of the linear network, whereas the points that determine the extremes of such segments are known as the vertices of the network.

Hence, a point process $X$ on $L$ is a finite point process in the plane (Diggle, 2013) such that all points of $X$ lie on the network $L$, whereas a collection of events that is observed on $L$ is known as a point pattern, $\boldsymbol{x}$, on $L$ (Ang et al., 2012; Baddeley et al., 2015, 2017). In particular, when every event of a point pattern has one or several attributes, the point pattern is referred to as a marked point pattern. Each of the attributes, which can be in the form of either a numerical or a categorical variable, is known as a mark of the pattern. The intensity function of a process on $L$, denoted by $\lambda(x)$, satisfies that the number $n(\boldsymbol{X} \cap B)$ of points of $\boldsymbol{X}$ falling in $B \subset L$ has expectation $\mathbb{E}[n(\boldsymbol{X} \cap B)]=\int_{B} \lambda(u) \mathrm{d}_{1} u$, where $d_{1} u$ denotes integration with respect to arc length (McSwiggan et al., 2017). Thus, given a point pattern, a typical objective is to estimate the intensity function of the process from which the locations of the events are assumed to be drawn, either through parametric or nonparametric techniques (Diggle, 
2013).

The investigation of spatial patterns lying on linear networks has gained attention in the last few years. The design of new and more accurate/efficient kernel density estimators (McSwiggan et al., 2017; Moradi et al., 2018, 2019; Rakshit et al., 2019b), the introduction of graph-related intensity measures (Eckardt and Mateu, 2018), the construction of local indicators of spatial association (Eckardt and Mateu, 2021), or the estimation of relative risks (McSwiggan et al., 2019) are some topics that have recently started to be developed for linear networks.

Besides the theoretical advances, it is worth noting that using linear networks for carrying out a spatial or spatio-temporal analysis entails certain technical difficulties. In this regard, the R package spatstat.linnet of the spatstat family (Baddeley et al., 2015) provides multiple specific functions that allow $\mathrm{R}$ users to carry out statistical analyses on linear networks. Furthermore, efforts are constantly being made to reduce the computational cost of adapting certain classical spatial techniques to the singular case of linear networks (Rakshit et al., 2019a).

Despite the existing necessity of analyzing point-referenced data coming from certain fields of research at the street level, there are not many software tools fully designed for hotspot detection on road networks. One relevant contribution in this regard is the KDE+ software (Bíl et al., 2016), but this is not integrated into R. The package DRHotNet is specifically prepared for allowing R users to detect differential risk hotspots (zones where a type of event is overrepresented) along a linear network. While the function relrisk. lpp from spatstat.linnet also allows $\mathrm{R}$ users to compute the spatially-varying probability of a type of event located in a linear network, DRHotNet provides a full procedure to detect, with high accuracy, the segments of a network where the probability of occurrence of a given type of event is considerably higher and optimize hotspot detection in terms of a prediction accuracy index widely used in criminology and other fields. There is no other R package currently providing this functionality.

\section{A procedure for detecting differential risk hotspots}

The procedure for differential risk hotspot detection available in DRHotNet was introduced by BrizRedón et al. (2019a), who also show an application of the method considering a traffic accident dataset. Overall, hotspot detection methods can be classified into partition-, hierarchy- and density-based methods (Deng et al., 2019). The one implemented in DRHotNet belongs to the last of these three groups.

Hence, the following subsections describe each of the steps that are carried out by the DRHotNet package. The specification and exemplification of the functions required for each of them are given in the following sections.

\section{Estimating a relative probability surface}

The first step consists in using kernel density estimation to infer the relative probability of occurrence for a certain type of event along a linear network. Given a marked point pattern $x=\left\{x_{1}, \ldots, x_{n}\right\}$, where a binary mark $y_{i}$ indicates if $x_{i}$ corresponds, or not, to the type of event of interest, the following expression is used to estimate this relative probability (Kelsall and Diggle, 1998):

$$
p_{h}(x)=\frac{\lambda_{h}^{\left\{x_{i}: y_{i}=1\right\}}(x)}{\lambda_{h}^{\left\{x_{1}, \ldots, x_{n}\right\}}(x)},
$$

where $\lambda_{h}^{\left\{x_{i}: y_{i}=1\right\}}(x)$ is an estimate of the intensity of the pattern formed by the events of interest (those that satisfy $y_{i}=1$ ) at location $x$, and $\lambda_{h}^{\left\{x_{1}, \ldots, x_{n}\right\}}(x)$ is an estimate of the intensity of the complete pattern at $x$. Both estimates are nonparametric and depend on a bandwidth parameter, $h$. Specifically, in DRHotNet package, $\lambda_{h}^{\left\{x_{i}: y_{i}=1\right\}}(x)$ and $\lambda_{h}^{\left\{x_{1}, \ldots, x_{n}\right\}}(x)$ are computed according to the network-constrained equal-split continuous kernel density estimation provided by McSwiggan et al. (2017). This version of kernel density is implemented in the function density . lpp of spatstat.linnet, which computes kernel density values rapidly by solving the classical heat equation defined on the linear network, as shown by McSwiggan et al. (2017). Despite the great performance of this approach, the computational cost of this version of kernel smoothing increases quadratically with $h$, so choosing very high values of $h$ can become too time-consuming.

Therefore, Equation 1 allows establishing a relative probability surface (referring to the type of event represented by $y_{i}=1$ ) on the linear network. To this end, given a location, $x$, of the linear network, the events which mainly contribute to the estimation of $p_{h}(x)$ are those situated within a linear radius (following the linear network structure) of $h$ meters from $x$. Thus, increasing the value of 
$h$ leads to smoother representations of the probability surface, whereas smaller values of the bandwidth parameter produce the opposite effect. On the choice of an optimal bandwidth parameter, the method described by McSwiggan et al. (2019) could be followed, which modifies previous proposals made for planar patterns (Kelsall and Diggle, 1995a,b).

Estimating a relative probability surface implies, in practice, estimating a relative probability value at the middle points of the segments forming the road network. In this regard, it is necessary to split the original road network structure into shorter line segments called lixels (Xie and Yan, 2008), which are analogous to pixels for a planar surface. This step provides accuracy and homogeneity to the process of hotspot formation. Then, the segments satisfying certain conditions (details are provided in the next section) are selected and joined together forming the differential risk hotspots.

\section{Determining differential risk hotspots}

Once a relative probability surface has been estimated along the linear network, it is time to detect differential risk hotspots. The procedure for their detection relies on two parameters $k$ and $n$. Parameter $k$ is used together with the standard deviation of all the relative probabilities estimated to define a threshold that needs to be exceeded to consider a segment as a candidate to be part of a differential risk hotspot. Then, parameter $n$ is employed to select the segments, from those satisfying the threshold condition, with $n$ or more events at a distance lower than $h$. More precisely, a segment, $i$, of the linear network is considered to be part of a differential risk hotspot if:

$$
\begin{aligned}
& \hat{p}_{i}>\operatorname{mean}\left(\left\{\hat{p}_{j}\right\}_{j=1}^{S}\right)+k \cdot S D\left(\left\{\hat{p}_{j}\right\}_{j=1}^{S}\right) \\
& \#\left\{x \in\left\{x_{1}, \ldots, x_{n}\right\}: d_{L}\left(x, m_{i}\right)<h\right\} \geq n,
\end{aligned}
$$

where $\hat{p}_{i}$ is the relative probability of occurrence for the type of event of interest estimated at the middle point of segment $i$, mean and $S D$ denote, respectively, the mean and the standard deviation of a finite set of numbers, $S$ is the number of segments of the linear network, \# denotes the cardinality of a finite set, $m_{i}$ is the middle point of segment $i$, and $d_{L}\left(x, m_{i}\right)$ represents the shortest-path distance (distance along the network) between $x$ and $m_{i}$.

The segments that fulfill the two conditions indicated above are then joined, forming differential risk hotspots. More precisely, two segments satisfying the aforementioned conditions belong to the same differential risk hotspot if they are neighbors. Given two segments of a linear network, a neighboring relationship exists between them if they share a vertex of the network, which is equivalent to the queen criterion used for defining polygon neighborhoods (Lloyd, 2010). This relationship between segments is referred to as a first-order one. Similarly, higher-order neighboring relationships are defined recursively (for instance, for a second-order relationship): $i$ and $j$ are second-order neighbors if one neighbor of $i$ and one neighbor of $j$ are first-order neighbors.

Note that choosing a higher value of either $k$ or $n$ implies being stricter in terms of determining differential risk segments. If the choice is too strict, segments that meet both conditions may not be found. One criterion for finding suitable values of $k$ and $n$ is to perform a sensitivity analysis on $k$ and $n$ (consisting of testing a set of plausible values for $k$ and $n$ ) and choosing a combination of both parameters that maximizes a measure that reflects the ability of the procedure to capture the overrepresentation of the event of interest along the network. These issues are clarified in subsequent sections.

\section{Measuring differential risk hotspots importance and significance}

\section{The prediction accuracy index}

Given a collection of hotspots in a planar surface, Chainey et al. (2008) defined the prediction accuracy index (PAI) as follows:

$$
\mathrm{PAI}=\frac{\text { Hit rate }}{\text { Area percentage }}=\frac{n / N}{a / A},
$$

where $n$ is the total number of events that lie on the hotspots, $N$ is the total number of events observed, $a$ is the area formed by all the hotspots together, and $A$ is the total area of the surface. Hence, a higher value of PAI is desirable in terms of hotspot detection as it represents a higher concentration of the events in relation to the size of the space they cover. This formula can be easily adapted to the case of linear networks using segment lengths in the place of areas.

In the context of detecting hotspots where the relative probability of one type of event is particularly high, the PAI needs to be modified. A type-specific version of the PAI (denoted by $\mathrm{PAI}_{t}$ ) was proposed 
in Briz-Redón et al. (2019a) (for linear networks):

$$
\mathrm{PAI}_{t}=\frac{n_{t} / N_{t}}{\ell / L}
$$

where $n_{t}$ is the number of events of the type of interest that lie on the hotspots, $N_{t}$ is the total number of events of that type that are observed, $\ell$ is the length of all the hotspots together, and $L$ is the total length of the network. The interpretation of the $\mathrm{PAI}_{t}$ is analogous to the one for the PAI. That is, a higher $\mathrm{PAI}_{t}$ value indicates a higher proportion of the type of interest compared to the proportion of road network length. Some researchers have recently explored the possibility of using the number of segments (in total and within the hotspots) instead of segment length (Drawve and Wooditch, 2019) in the denominator of this formula, but we opted for segment length proportions.

It is also worth noting that the $\mathrm{PAI}_{t}$ can be computed for the whole set of differential risk hotspots detected, as indicated above, or for each differential risk hotspot individually. The first option is useful for comparing the level of spatial concentration that two or more types of events show along the network or for assessing the efficiency of different hotspot detection procedures. The second option is suitable for determining which differential risk hotspot maximizes the quotient between the proportion of the type and the proportion of road length spanned, which may represent the importance of the hotspot.

\section{Estimating a $p$-value}

Finally, assigning a statistical significance value to each differential risk hotspot is necessary to avoid the possibility of focusing on certain microzones of the network that do not deserve such attention. Thus, a Monte Carlo approach was used to estimate an empirical $p$-value for each differential risk hotspot yielded by the previous step of the procedure. This approach is similar in spirit to the one proposed by Bíl et al. (2013), which is applied in the KDE+ software mentioned before.

Getting back to previous notation, if $\left\{x_{1}, \ldots, x_{n}\right\}$ is a marked point pattern, and $y_{i}$ a binary mark that indicates if $x_{i}$ corresponds, or not, to the type of event under analysis, the Monte Carlo approach implemented consists in generating $K$ simulated datasets where the locations are left fixed and the marks permutated. Concretely, this means to keep the locations $\left\{x_{1}, \ldots, x_{n}\right\}$ and to obtain a new collection of marks defined by $y_{i}^{k}=y_{\rho(i)}$, where $k$ indicates the iteration number $(k=1, \ldots, K)$, and $\rho$ is a permutation of the first $n$ natural numbers. For each simulation, the average relative probability presented by each differential risk hotspot (computed as the weighted average per segment length of the relative probabilities estimated at the middle points of the segments composing the hotspot) is obtained and denoted by $\hat{s}_{k}(k=1, \ldots, K)$. Therefore, if $\hat{r}$ represents such average relative probability for a hotspot, considering the original dataset, the rank of $\hat{r}$ within the ordered vector formed by the $\hat{s}_{k}$ 's and $\hat{r}$ allows estimating an empirical $p$-value for the corresponding hotspot:

$$
p=1-\frac{\#\left\{k \in\{1, \ldots, K\}: \hat{s}_{k} \leq \hat{r}\right\}}{K+1}
$$

\section{Dealing with linear networks in $\mathbf{R}$}

\section{Classes and functions}

Linear networks can be represented in $\mathrm{R}$ by the class SpatialLines of package sp (Pebesma and Bivand, 2005; Bivand et al., 2013) or by simple features with packages sf (Pebesma, 2018) and sfnetworks (van der Meer et al., 2021). However, the class linnet from the spatstat.linnet package is optimal for doing spatial analysis and modeling on linear networks.

There are several functions in $\mathrm{R}$ that facilitate the conversion between Spatial Lines and linnet objects. Specifically, as . linnet. SpatialLines from the maptools (Bivand and Lewin-Koh, 2017) $\mathrm{R}$ package converts Spatiallines into linnet objects, whereas the double application (in this order) of the as.psp and as.Spatiallines.psp functions of the spatstat.geom and maptools packages, respectively, enable the conversion from a linnet object into a Spatiallines one.

Other useful functions available in spatstat.linnet for the use of linear networks are, for example, connected. I innet (computes the connected components of a linear network), diameter. linnet (computes the diameter and bounding radius of a linear network), insertVertices (inserts new vertices in a linear network), and thinNetwork (removes vertices or segments from a linear network). 


\section{Preprocessing the linear network}

Although not strictly necessary, preprocessing the linear network is convenient to facilitate the subsequent statistical analysis. The SpNetPrep package (Briz-Redón, 2019) can be used for this purpose. The manual edition of the network, the addition of directionality, and the curation of a point pattern lying on a network can be performed through a Shiny-based application implemented in this package if the linear network represents a road network (which is the most common scenario and the one we assume for the example shown in this paper). Furthermore, SpNetPrep contains a function, SimplifyLinearNetwork, that allows users to reduce the network's complexity by merging some pairs of edges of the network that meet certain conditions on their length and angle. In this regard, another option is to use the gSimplify function of rgeos (Bivand and Rundel, 2020), which provides an implementation of the Douglas-Peuker algorithm for curve simplification (Douglas and Peucker, 1973).

\section{Creating a point pattern on a linear network}

Function lpp of package spatstat.linnet can be used to create an $\mathrm{R}$ object that represents a point pattern lying on a linear network. The lpp function only requires the coordinates of the events and a linnet object corresponding to a linear network. For instance, the following commands can be typed to create a point pattern of 100 points over the simplenet network provided by spatstat.data, which lies within the $[0,1] \times[0,1]$ window:

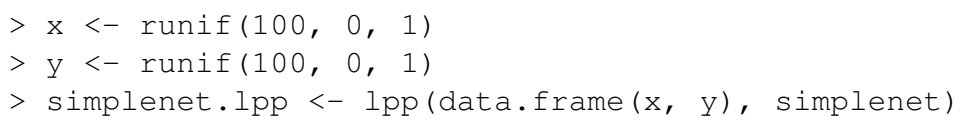

Marks can be attached to the points forming the pattern by introducing several more columns next to the $\mathrm{x}$ and $\mathrm{y}$ coordinates. For example, one can introduce a continuous random mark following a standard normal distribution or a categorical random mark.

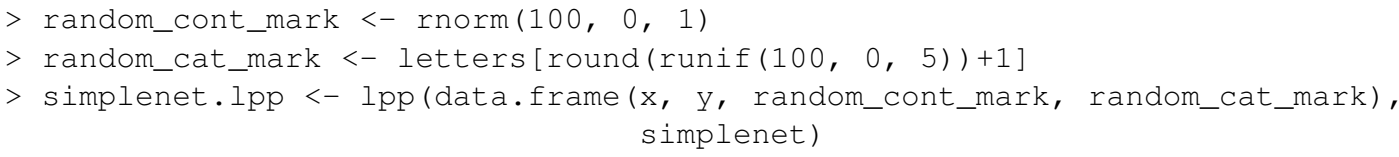

In order to fit the objective of computing a relative probability for one type of event, categorical marks are required. However, recoding a continuous mark into several categories to facilitate the estimation of a specific relative risk is one possible alternative.

\section{Using DRHotNet}

This section shows the complete use of the DRHotNet package with a dataset of crime events recorded in Chicago (Illinois, US). The reader should note that some of the outputs may vary slightly due to possible changes over time in some of the packages used. First of all, the following R libraries have to be loaded to reproduce the example:

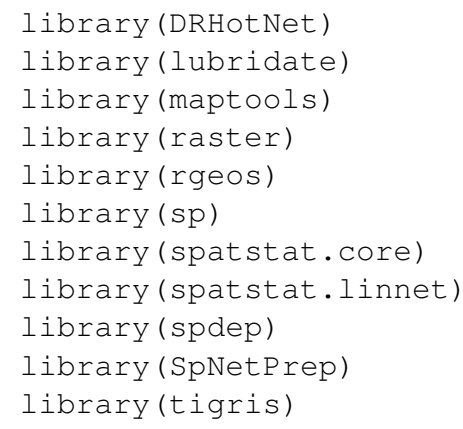

\section{Downloading and preparing the linear network}

The examples provided in this section fully employ open data available for Chicago. First, geographic data from Chicago was downloaded from the United States Census Bureau through package tigris (Walker, 2016). Specifically, census tracts and the road network of the state of Massachusetts were 
loaded into the R console. The function intersect from the package raster (Hijmans, 2019) can be used.

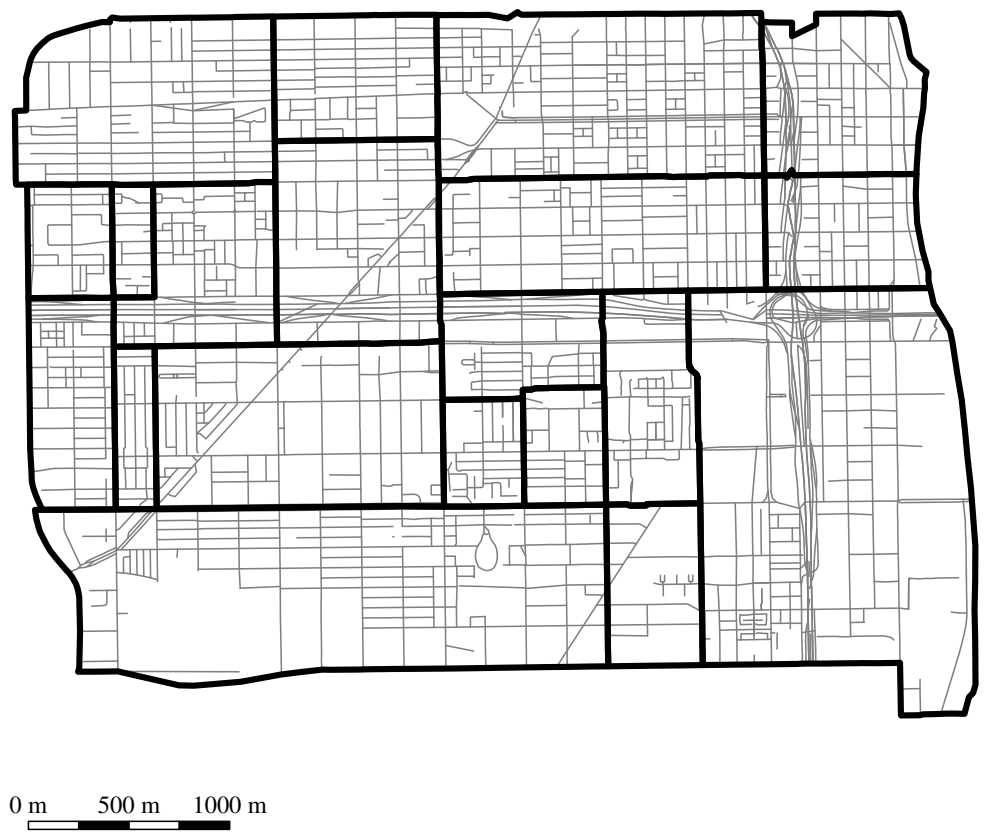

Figure 1: Road network (in gray) corresponding to the Near West Side Community Area of Chicago. Census tracts of the area are overlayed in black.

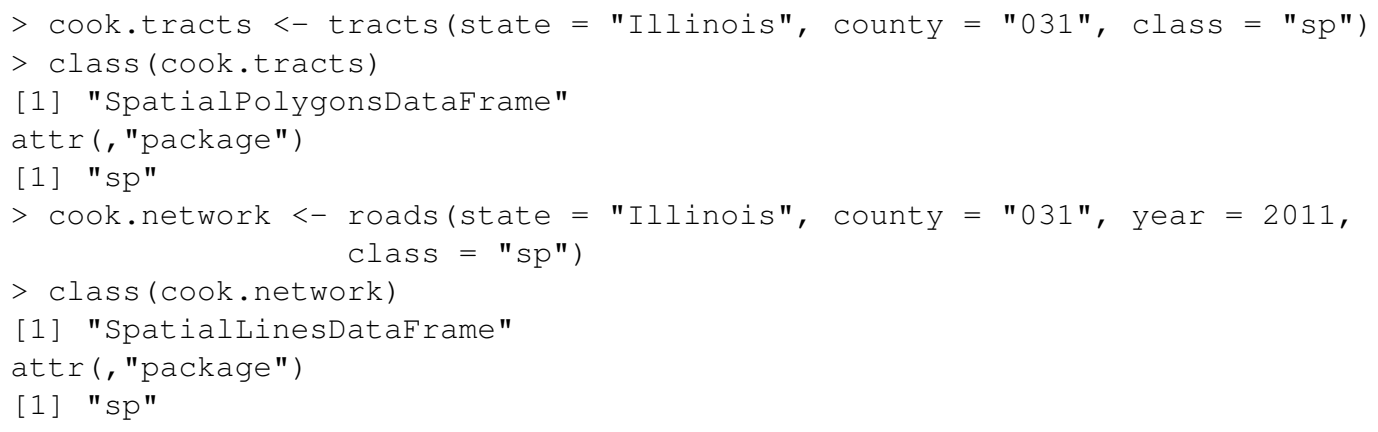

The objects cook. tracts and cook . network are composed of 1319 polygons and 86227 lines, respectively (as of the end of June 2021). Now, both spatial objects are intersected to construct a smaller road network that corresponds to the Near West Side Community Area of Chicago.

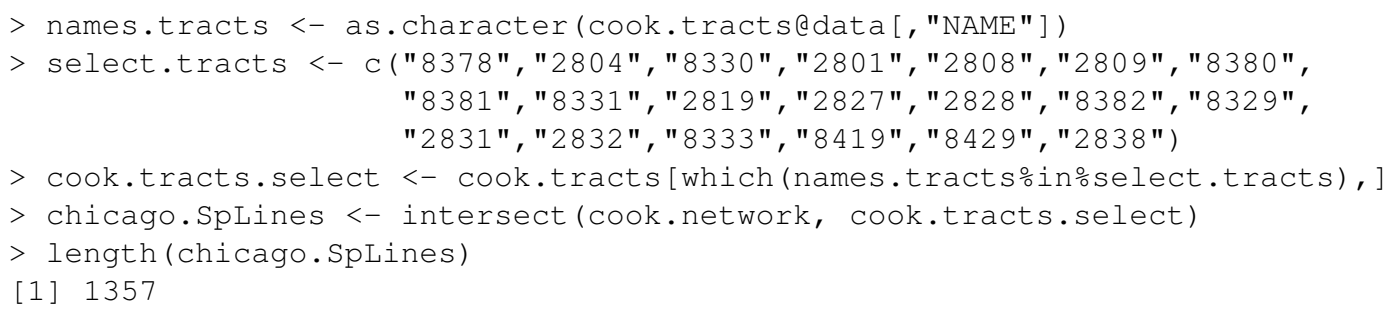

Object chicago. Spline (SpatialLinesDataFrame) has 1357 lines. Then, this object's coordinates are converted into UTM (Chicago's UTM zone is 16):

$>$ chicago.Splines <- spTransform(chicago.SpLines,

"+proj=utm +zone=16 ellps=WGS $84 "$ ) 
Now the corresponding linnet object is created:

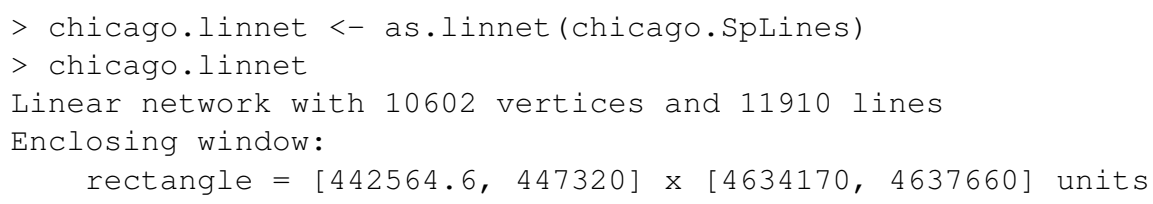

It is worth noting how the transformation of the network into a linnet object increases dramatically the number of line segments (from 1357 to 11910). This is a consequence of the fact that SpatialLines objects can handle curvilinear segments, made of multiple line segments, as a single line. However, l innet objects follow the definition of the linear network provided in the Introduction section, which excludes this possibility.

It is required that the network is fully connected to allow the computation of a distance between any pair of points. This can be checked with the function connected.

$>$ table (connected (chicago.linnet, what = "labels"))

$\begin{array}{rrrrrrrrr}1 & 2 & 3 & 4 & 5 & 6 & 7 & 8 & 9 \\ 10575 & 5 & 2 & 2 & 2 & 5 & 2 & 2 & 7\end{array}$

This output indicates that there is a connected component of 10575 vertices and other eight components with only a few vertices. The use of connected with the option what = "components" enables us to extract the larger connected component for the analysis, discarding the others.

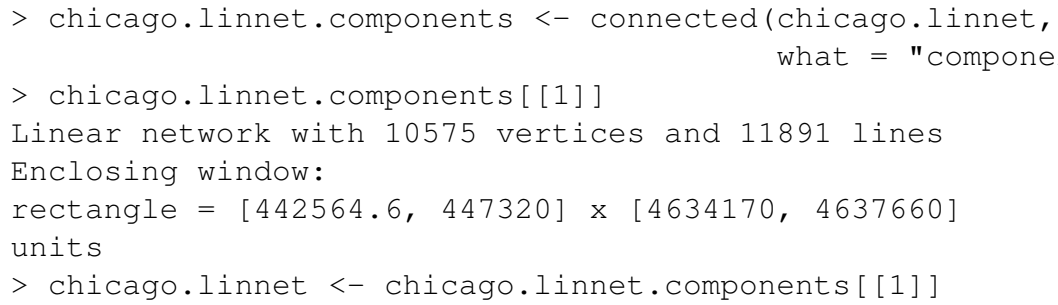

At this point, it is worth considering the possibility of reducing the network's complexity. The function SimplifyLinearNetwork of SpNetPrep can be used for this purpose. A reasonable choice of the parameters is Angle $=20$ and Length $=50$ (Briz-Redón, 2019). This choice of the parameters means that a pair of segments meeting at a second-degree vertex are merged into one single segment if the angle they form (measured from $0^{\circ}$ to $90^{\circ}$ ) is lower than $20^{\circ}$ and if the length of each of them is lower than $50 \mathrm{~m}$. Hence, the network's complexity is reduced (in terms of the number of segments and lines) while its geometry is preserved. The following lines of code execute SimplifyLinearNetwork and redefine chicago. SpLine according to the structure of the final linnet object.

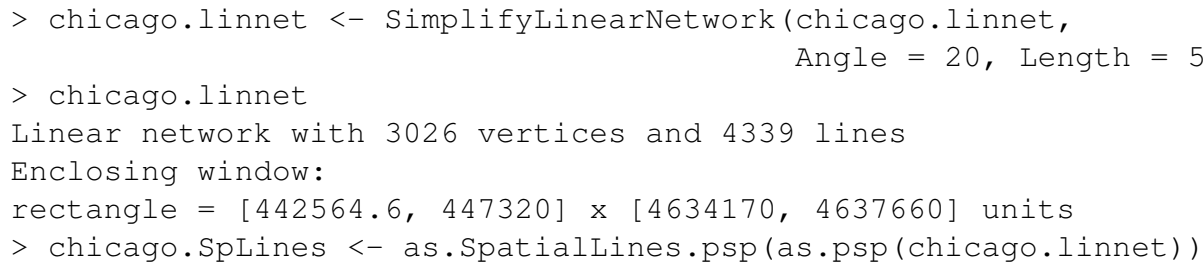

Thus, the final road network from Chicago that we use for the analysis has 4339 lines and 3026 vertices. An example of how SimplifyLinearNetwork reduces the network's complexity is shown in Figure 2, which corresponds to a squared zone of Chicago's network with a diameter of $600 \mathrm{~m}$.

\section{Downloading and preparing crime data}

Point-referenced crime datasets corresponding to several cities from the United States of America can be downloaded through the R package crimedata (Ashby, 2019). Concretely, crimedata currently provides (as of June 2021) crime open data recorded in Austin, Boston, Chicago, Detroit, Fort Worth, Kansas City, Los Angeles, Louisville, Mesa, New York, San Francisco, Tucson, and Virginia Beach. Therefore, the function get_crime_data from this package can be used for downloading a dataset of crime events recorded in Chicago in the period 2007-2018. 


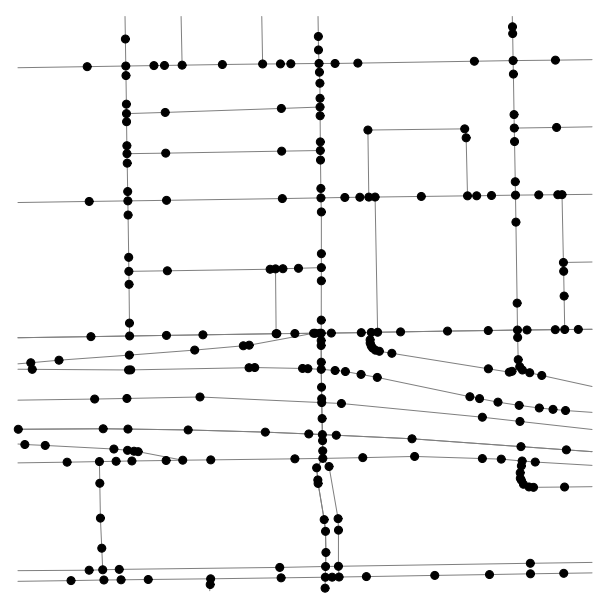

(a)

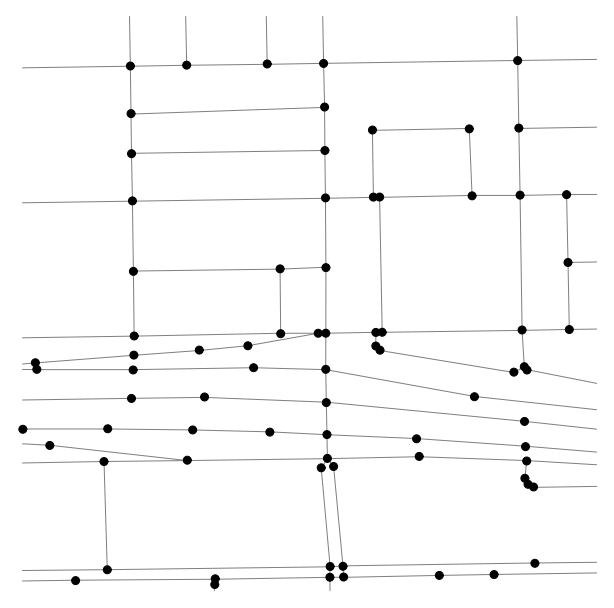

(b)

Figure 2: Extracting a part of the road network structure analyzed from Chicago. Original structure extracted (left), made of 285 lines and 272 vertices, and simplified version of it (right) with 122 lines and 109 vertices.

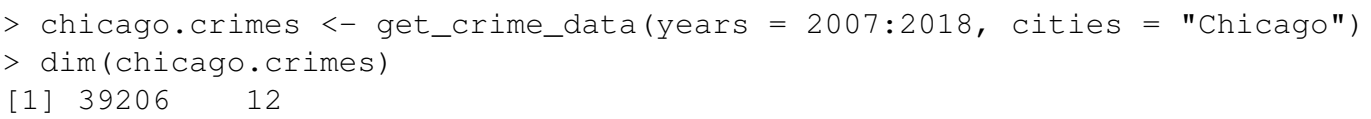

The year, month, and hour of occurrence of each crime can be extracted with the corresponding functions of the package lubridate (Grolemund and Wickham, 2011).

$>$ chicago.crimes\$year <- year (chicago.crimes\$date_single)
$>$ chicago.crimes\$month <- month (chicago.crimes\$date_single)
$>$ chicago.crimes\$hour <- hour (chicago.crimes\$date_single)

Then, a marked point pattern lying on chicago. linnet can be created with function lpp to provide the framework required by the DRHotNet package. A data. frame is passed to lpp including the coordinates of the events (in UTM), the type of event according to the receiver of the offense (offense_against), and the year, month, and hour of occurrence that have been just computed.

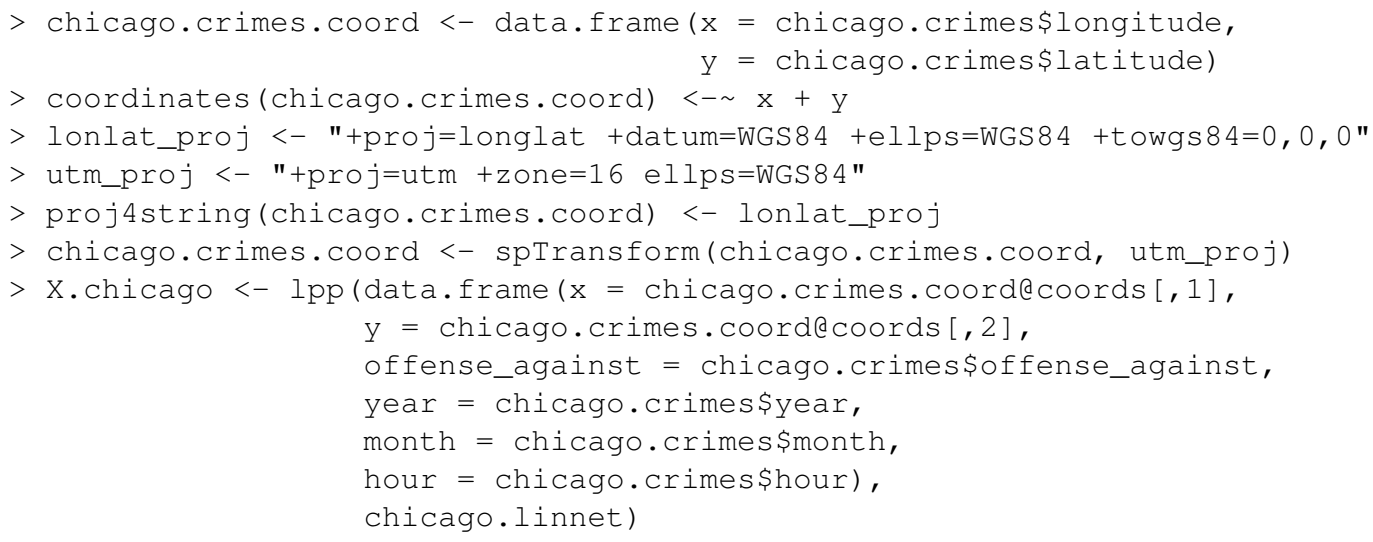

Warning message:

38006 points were rejected as lying outside the specified window

A total of 38006 points are rejected because they lie outside the road network. Hence, a marked point pattern of 1200 crimes that lie on chicago. linnet remains for the analysis. The four marks are categorical, presenting the following values and absolute frequencies:

\footnotetext{
$>$ table(X.chicago\$data\$offense_against)

other persons property society
} 


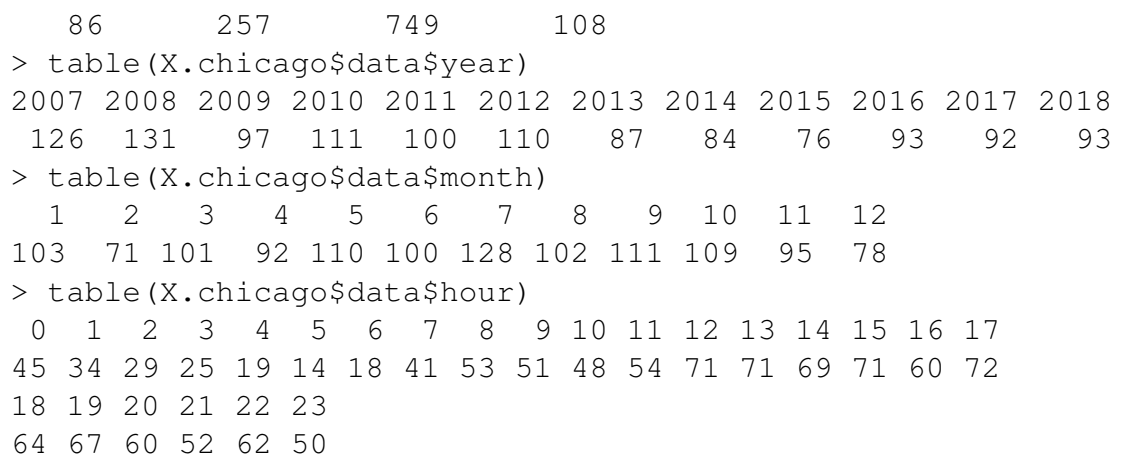

Hence, DRHotNet functionalities are now applicable to X. chicago. In the main example shown, the relative occurrence of crimes against persons along the road network included in X. chicago is analyzed. To this end, the functions of the package are used following the steps that have been established for the differential risk hotspot detection methodology previously described.

\section{Estimating a relative probability surface}

The function relpnet of DRHotNet has to be used at first to estimate a relative probability surface over the linear network. As it has been explained, this implies estimating a relative probability in the middle point of each segment of the network.

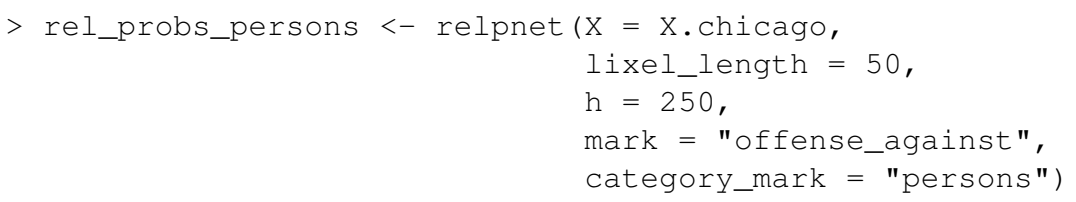

The available parameter finespacing is by default set to FALSE in order to reduce the computational cost (as FALSE is the default value, it can be omitted in the specification of the function). If $f$ inespacing is set to TRUE, more accurate kernel density estimates are obtained at short segments, but the computational cost can become prohibitive. The interested reader can consult the documentation of the densityHeat function of spatstat.linnet to know precisely how this parameter works.

In this example, an upper bound of $50 \mathrm{~m}$ is chosen for the lixel length. This means that segments shorter than $50 \mathrm{~m}$ are not split, whereas those longer than $50 \mathrm{~m}$ are split into several shorter lixels of no more than $50 \mathrm{~m}$ lengths. This operation is performed internally by relpnet with the function lixellate from spatstat.linnet. The bandwidth parameter, $h$, is set to $250 \mathrm{~m}$. The mark and category_mark parameters are used to specify the type of event that is under analysis.

The exploration of the object rel_probs_persons with function str allows the user to check that the choice of a $50 \mathrm{~m}$ threshold for the lixel length produces 8617 segments along the network. Thus, a relative probability is estimated in the middle point of each of these segments, which can be accessed by typing \$probs:

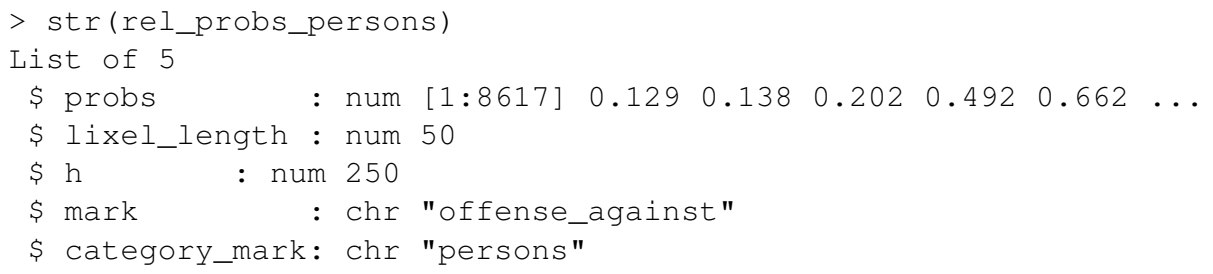

The function plotrelp can then be used to obtain a map of the relative probability surface like the one shown in Figure 3b. Figure 3 also contains the relative probability surfaces corresponding to the choices of $h=125$ (Figure 3a), $h=500$ (Figure 3c) and $h=1000$ (Figure 3d). In this particular case, using an Intel(R) Core(TM) i7-6700HQ processor, it takes approximately 1 second to execute relpnet if $h=125$, and 8 seconds if $h=1000$.

Figure 1 allows us to observe that the choice of a larger value for $h$ smooths the relative probability surface, which in the case of $h=500$ or $h=1000$ leads to the configuration of a small number of clearly distinguishable zones of the network in terms of the relative probability of offenses against persons. Indeed, whereas the use of $h=125$ allows the user to obtain quite extreme relative probability values 
Relative probabilities 'persons' (offense_against), lixel_length $=50, h=125$
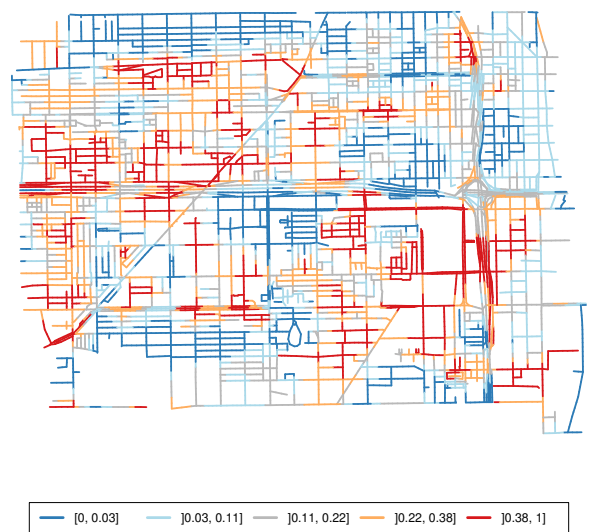

(a)

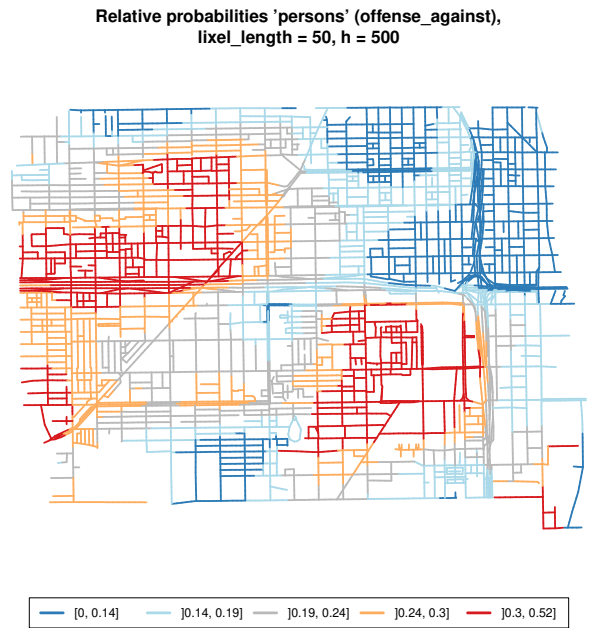

(c)
Relative probabilities 'persons' (offense_against), lixel_length $=50, h=250$
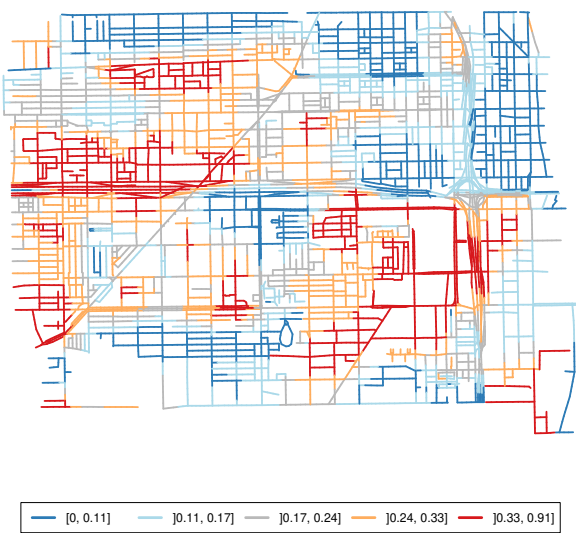

(b)

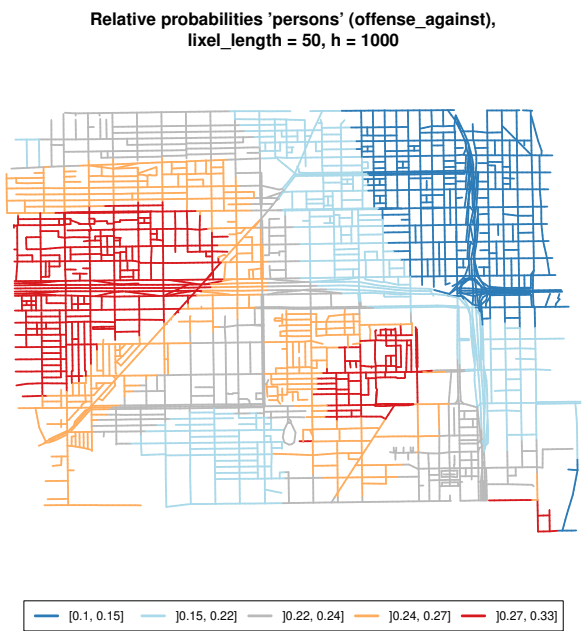

(d)

Figure 3: Outputs from the function plotrelp for the following choices of $h$ : 125 (a), 250 (b), 500 (c) and $1000(\mathrm{~d})$.

at some segments of the network (the relative probability estimates cover the [0,1] interval), choosing $h=1000$ causes that all the relative probabilities lie in the interval [0.10,0.33].

In view of Figure 3, we consider that $h=250$ is a reasonable choice (although some procedures for bandwidth selection could be explored for taking this decision). Therefore, this selection of the bandwidth parameter is maintained to display now how the drhot function of the package works.

\section{Detecting differential risk hotspots}

The function drhot needs four parameters to be specified: $\mathrm{x}$ (a point pattern on a linear network), rel_probs (an object like the one obtained in the previous step), k, and n. Parameters $k$ and n control the differential risk hotspot procedure, as it has been explained before. For example, we can try with $\mathrm{k}$ $=1$ and $\mathrm{n}=30$ :

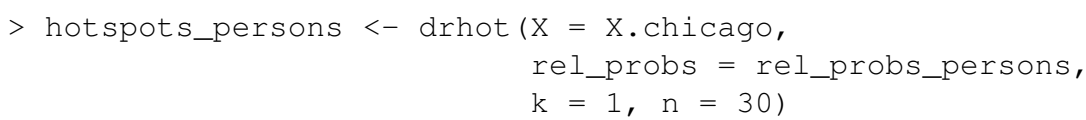

The output of the function drhot presents the following structure:

$>\operatorname{str}($ hotspots_persons)

List of 8 


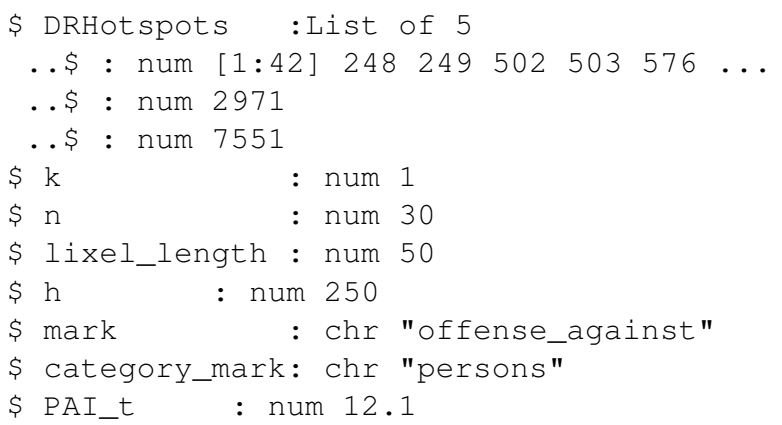

The first component of hotspots_persons contains the differential risk hotspots that have been detected for the values of $k$ and $n$ provided to drhot. In this case, three differential risk hotspots are found, which are formed by 42,1 , and 1 road segments, respectively. The object hot spots_persons also contains the values of the parameters involved in the computation of the hotspots and a final component that includes the global $\mathrm{PAI}_{t}$ for the set, which is 12.10 in this example.

The function drsummary can be used to provide a summary of each of the differential risk hotspots determined by drhot:

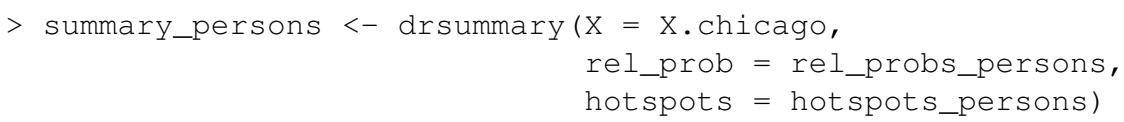

The output of drsummary includes a count of the number of events located within each differential risk hotspot and how many of these correspond to the category "persons":

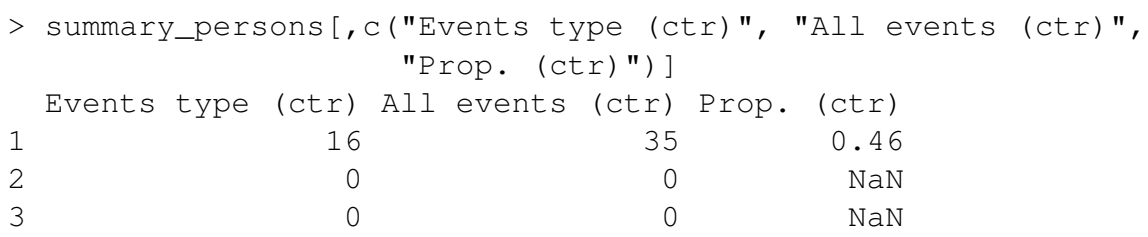

The summary also contains the length (in meters) of each differential risk hotspot and the $\mathrm{PAI}_{t}$ that corresponds to each of them:

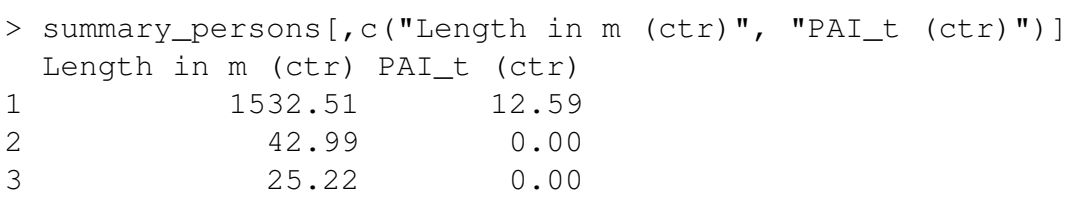

Furthermore, the output of drsummary also provides the same statistics for an extension of each of the hotspots. The reason to include this information is that if there are not many events available in the dataset (as it happens in this example), the method can determine differential risk hotspots where very few events, if any, have taken place. Indeed, in the output of drsummary shown above, there are two hotspots including zero events. The fact of employing kernel density estimation to infer a relative probability surface makes it more convenient to think of each differential risk hotspot as the union of a center or core (what drhot returns, the hotspot itself) and an extension of it. Hence, by considering an extension of the differential risk hotspot, one can better appreciate the zone of the network that has been accounted for in the estimation of the relative probability values corresponding to the segments of the hotspot.

By default, the extension computed by drsummary coincides with a neighbourhood of the seg-

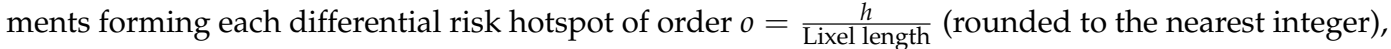
although a different order can be specified through the parameter order_extension. In this example, we have $o=\frac{250}{50}=5$, which is used by drsummary if no other order is indicated:

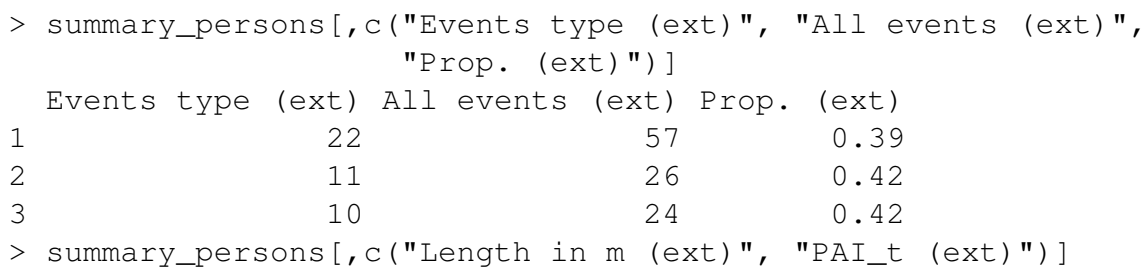




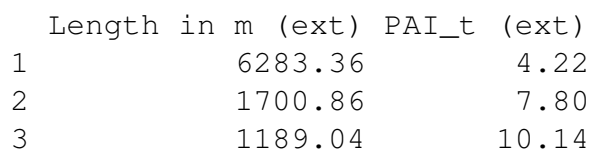

It can be observed that all extensions of the differential risk hotspots include a reasonable number of events and that the corresponding proportions of offenses against persons are clearly above the global proportion for the dataset, which is $257 / 1200=21.42$.

\section{Assessing the statistical significance of the hotspots}

Following the choice of $\mathrm{k}=1$ and $\mathrm{n}=30$, it only remains to estimate a $p$-value for each differential risk hotspot detected. This can be done by calling the function drsummary again and specifying compute_p_value $=\mathrm{T}$. A total of 20 iterations are selected for performing the Monte Carlo simulation process:

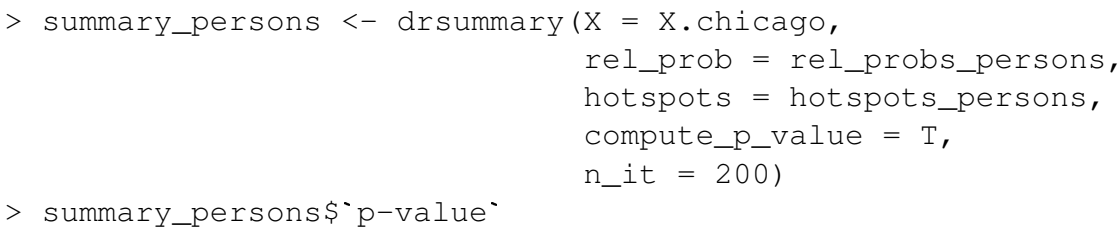

Therefore, the five differential risk hotspots detected with $k=1$ and $n=30$ are statistically significant $(p<0.05)$. It is worth noting, however, that the usual significance level of 0.05 should be reduced (corrected) if many differential risk hotspots are detected to avoid the presence of multiple comparison problems.

\section{Choosing $k$ and $n$}

Remember that a higher value of either $\mathrm{k}$ or $\mathrm{n}$ represents using a more stringent criterion regarding hotspot detection. This is illustrated through the four maps available in Figure 4, which can be generated with the function plothot of DRHotNet. For instance, as in the following example for the object hot spots created previously (which corresponds to Figure $4 \mathrm{~d}$ ):

$>$ plothot $(\mathrm{X}=\mathrm{X}$.chicago, hotspots $=$ hotspots_persons $)$

Indeed, Figure 4 shows the differential risk hotspots that drhot detects for the choices of $\mathrm{k}=$ 0.5 and $\mathrm{n}=20$ (Figure $4 \mathrm{a}$ ), $\mathrm{k}=1.5$ and $\mathrm{n}=20$ (Figure $4 \mathrm{~b}$ ), $\mathrm{k}=1$ and $\mathrm{n}=10$ (Figure $4 \mathrm{c}$ ), and $\mathrm{k}=1$ and $\mathrm{n}=30$ (Figure $4 \mathrm{~d}$ ). Two of these combinations of conditions on $k$ and $n$ are implicitly represented by the other two. Consequently, the differential risk hotspots shown in Figure $4 \mathrm{~b}$ are contained in Figure 4a, and those in Figure $4 \mathrm{~d}$ are contained in Figure 4c. The choice of $\mathrm{k}=1$ and $\mathrm{n}$ $=30$ leads to the highest global $\mathrm{PAI}_{t}$ among the four combinations of parameters indicated with the value of 12.1 mentioned before. In this regard, we recommend performing a sensitivity analysis on the values of $k$ and $n$ to decide which combination is more convenient. The sensitivity analysis carried out by Briz-Redón et al. (2019a) yielded that a choice around $k=1.5$ and $n=45$ was optimal in terms of the $\mathrm{PAI}_{t}$ for the traffic accident dataset that was investigated. However, each dataset should require a specific analysis.

Thus, a sensitivity analysis on $k$ and $n$ can be carried out with the function drsens. The user has to provide a point pattern (X), an object containing the relative probabilities of a type of event along the network (rel_probs) and a set of values for $k$ and $n$ (ks and ns, respectively):

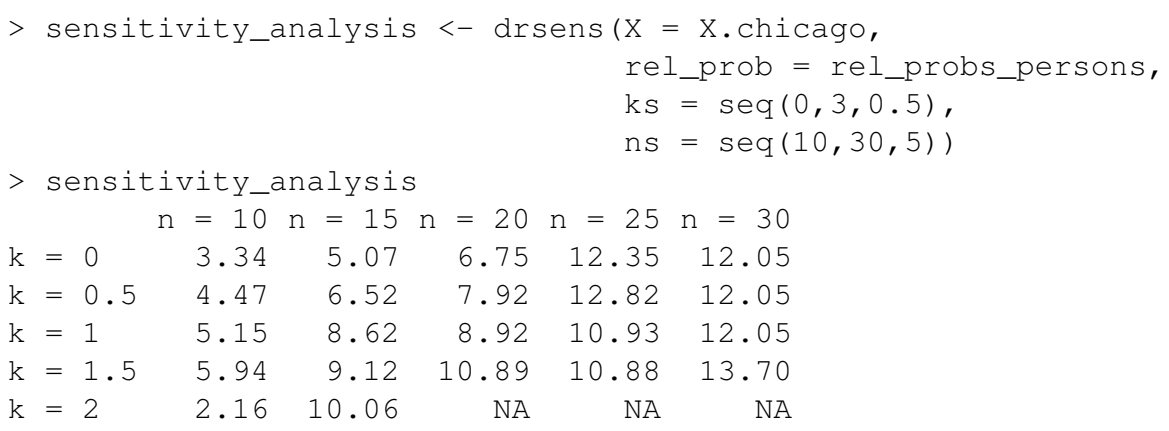




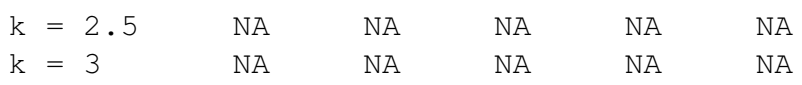

The output from drsens is a matrix that contains the $\mathrm{PAI}_{t}$ values that correspond to each combination of $k$ and $n$ indicated by ks and ns. A NA value represents that no differential risk hotspots can be found for such a combination of parameters. According to this matrix, the highest $\mathrm{PAI}_{t}$ value is achieved for $k=1.5$ and $n=30$.

Therefore, one can choose the values of $k$ and $n$ that maximize the $\mathrm{PAI}_{t}$ (considering the parameters provided in ks and ns) to determine the final set of differential risk hotspots. However, this criteria may sometimes lead to detecting a very low number of differential risks hotspots and hence to miss zones of the network that may also deserve some attention. Hence, a more conservative approach could be considering several combinations of $k$ and $n$ that yield some of the highest values of PAI $t$ and explore each set of differential risk hotspots associated. Then, one could investigate the output of drsummary for each combination of parameters (including the computation of $p$-values) to better decide which zones of the network are relevant for the type of event of interest.
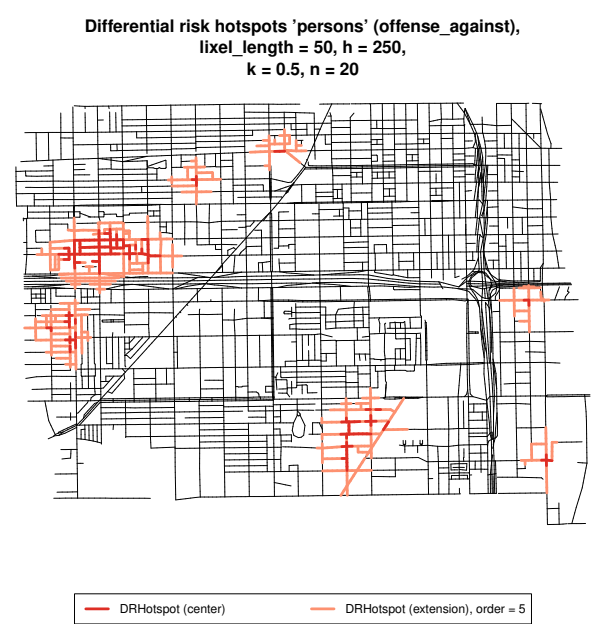

(a)

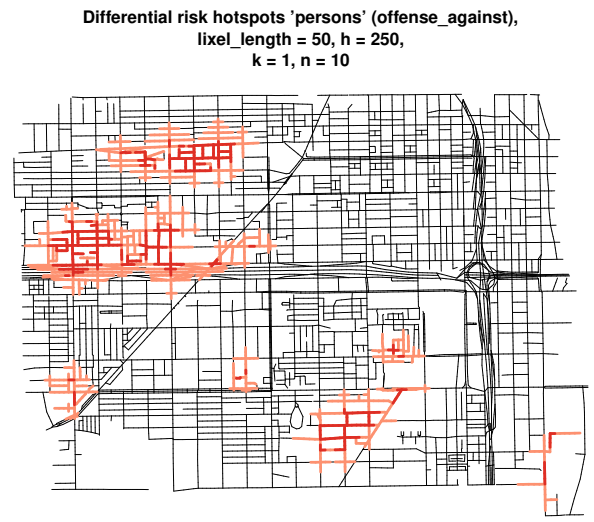

- DRHotspot (center)
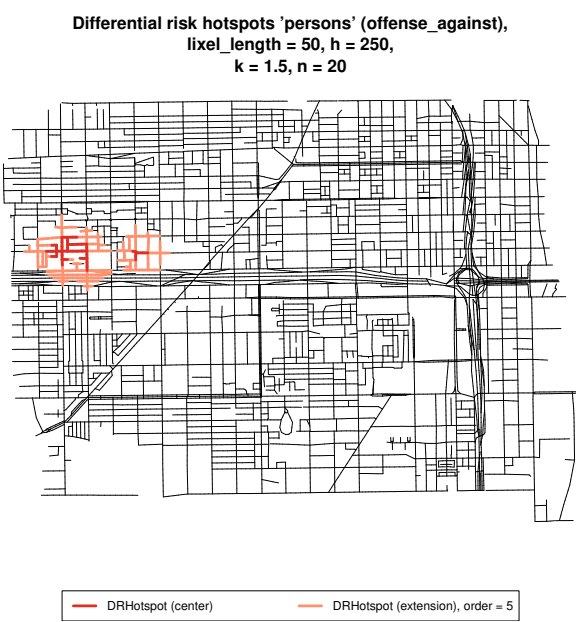

(b)

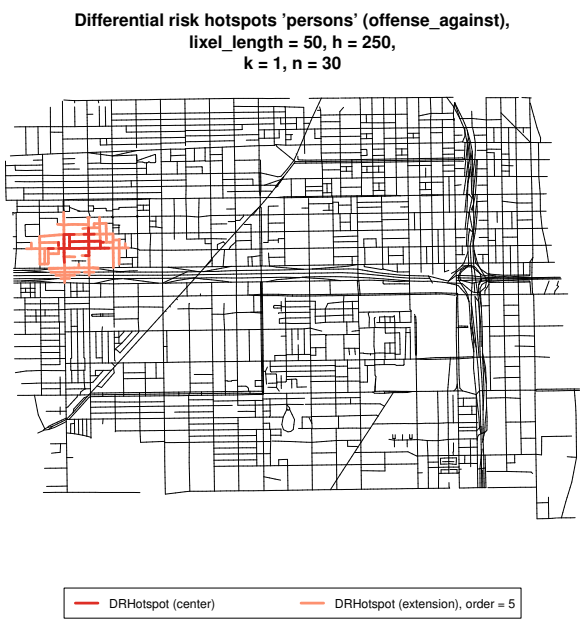

(d)

Figure 4: Outputs from the function plothot for the following choices of $k$ and $n: k=0.5$ and $n=20$ (a), $k=1.5$ and $n=20(\mathrm{~b}), k=1$ and $n=10$ (c), and $k=1$ and $n=30$ (d).

\section{Other applications of the methodology}

This final section shows the results that are obtained for other type of events for comparative purposes. First, the marks X. chicago are recoded into binary outcomes as follows: 
> year_after_2012 <- ifelse (X.chicago\$data\$year>2012, "Yes", "No")

$>$ month_winter <- ifelse (X.chicago\$data\$month\%in\%c $(12,1,2)$, "Yes", "No")

$>$ hour_21_3 <- ifelse (X.chicago\$data\$houroinoc $(21: 23,0: 3)$, "Yes", "No")

$>$ marks(X.chicago) <- data.frame (as.data.frame (marks (X.chicago)),

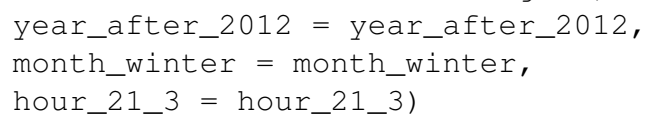

The relative probability surfaces have to be computed. We used the same values for lixel. length and $h$ than in the previous examples.

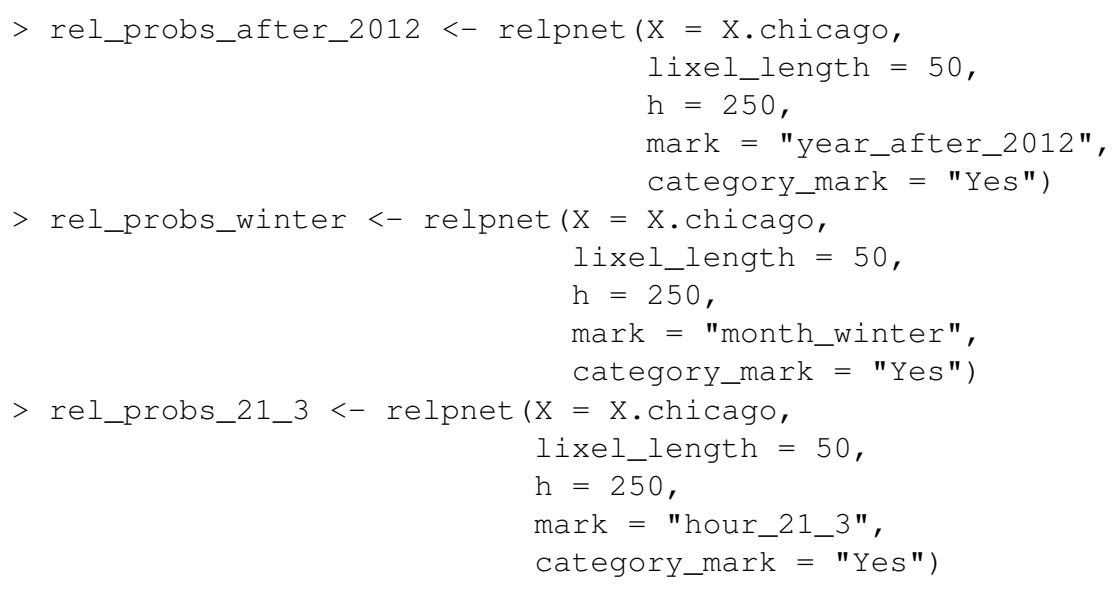

The corresponding sensitivity analyses are carried out:

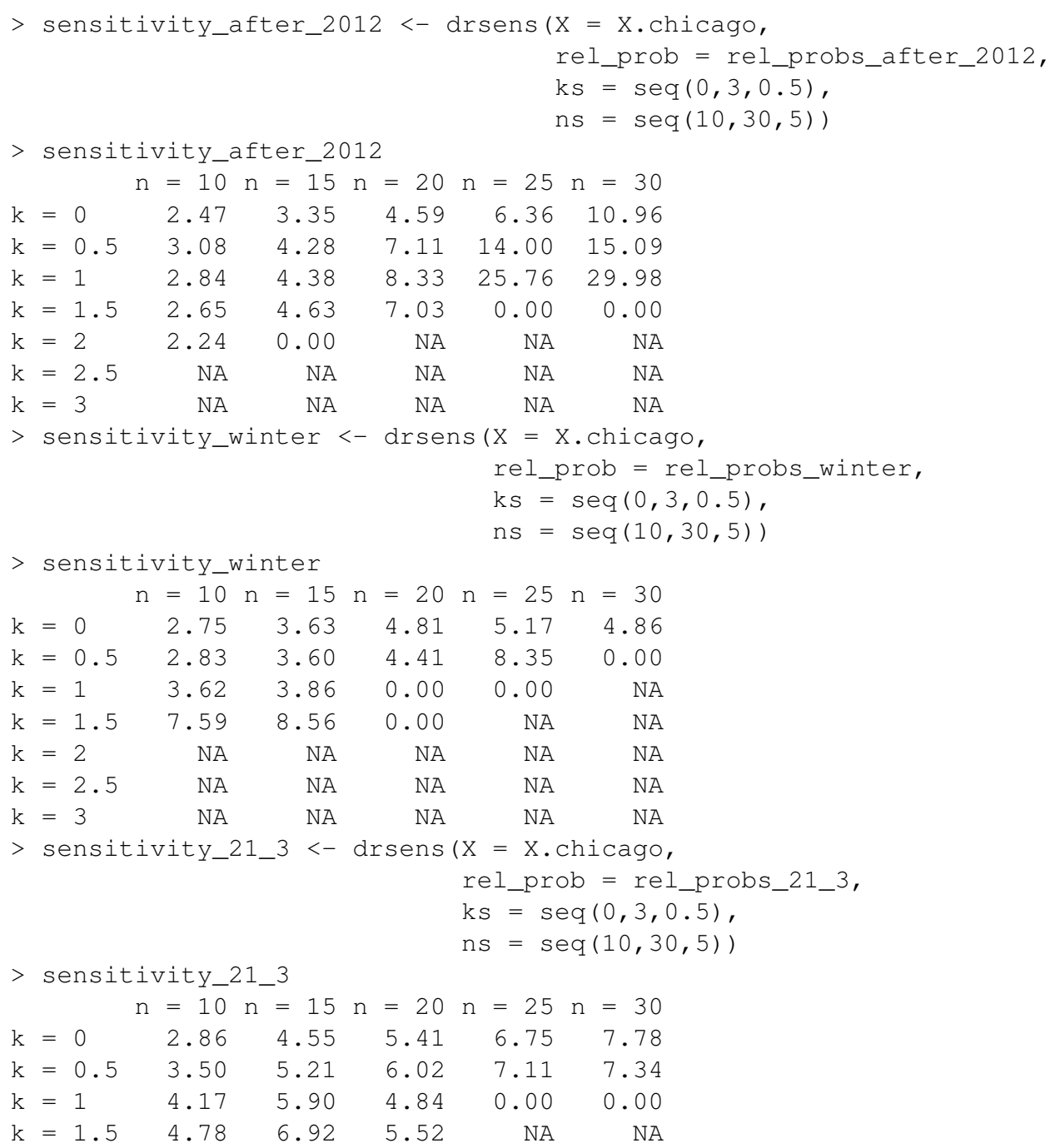




$\begin{array}{lrrrrr}\mathrm{k}=2 & 5.14 & 5.64 & 3.00 & \mathrm{NA} & \mathrm{NA} \\ \mathrm{k}=2.5 & 4.59 & 1.75 & 0.00 & \mathrm{NA} & \mathrm{NA} \\ \mathrm{k}=3 & \mathrm{NA} & \mathrm{NA} & \mathrm{NA} & \mathrm{NA} & \mathrm{NA}\end{array}$

The highest $\mathrm{PAI}_{t}$ values for year_after_2012, month_winter, and hour_21_3 are 29.98, 8.56, and 7.78, respectively. The differential risk hotspots that are obtained for the combination of $k$ and $n$ that lead to these $\mathrm{PAI}_{t}$ values can be visualized with plot hot (Figure 5):

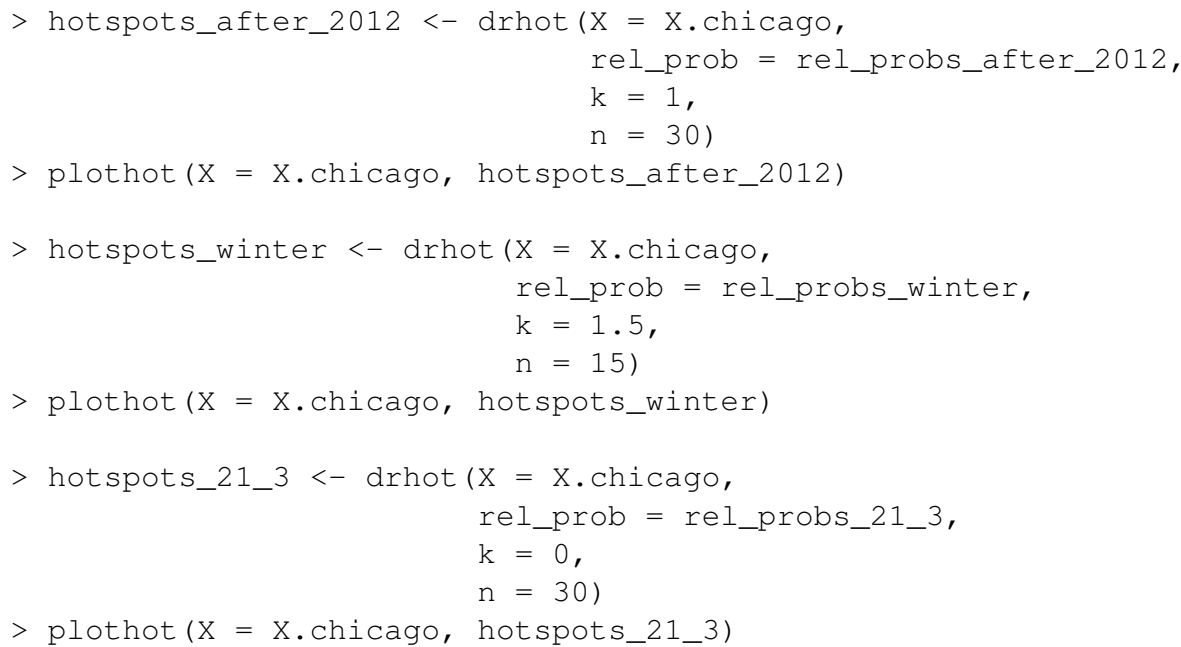

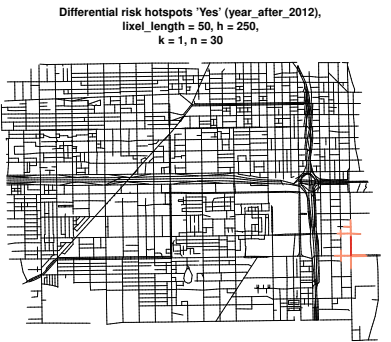

(a)

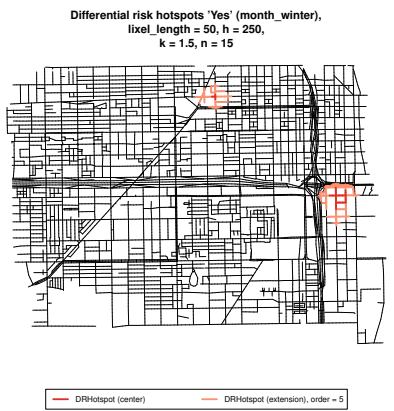

(b)

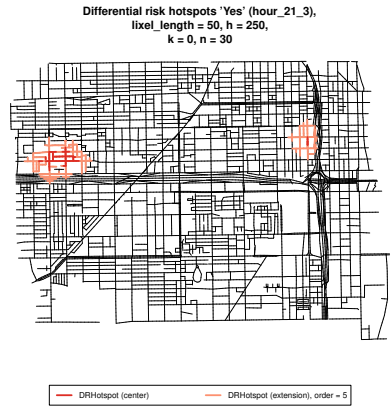

(c)

Figure 5: Outputs from the function plothot for the marks year_after_2012, month_winter, and hour_21_3 and the categorical value Yes for the three, considering the combinations of $k$ and $n$ that maximize the $\mathrm{PAI}_{t}$ (for the values of $k$ and $n$ tested).

\section{Summary}

The R package DRHotNet for detecting differential risk hotspots on linear networks has been described. The use of linear networks in the context of hotspot detection is becoming more important over the years, particularly in the fields of criminology and traffic safety. In addition, it is also of great interest sometimes to detect zones of a linear network where a certain type of event is especially overrepresented. Hence, DRHotNet consists of an easy-to-use tool implemented in R to accurately locate the microzones of a linear network where the incidence of a type of event is considerably higher than in the rest of it.

\section{Bibliography}

B. Allévius. scanstatistics: Space-time anomaly detection using scan statistics. The Journal of Open Source Software, 3:515, 2018. [p380] 
M. A. Andresen, A. S. Curman, and S. J. Linning. The trajectories of crime at places: understanding the patterns of disaggregated crime types. Journal of Quantitative Criminology, 33(3):427-449, 2017. [p380]

Q. W. Ang, A. Baddeley, and G. Nair. Geometrically corrected second order analysis of events on a linear network, with applications to ecology and criminology. Scandinavian Journal of Statistics, 39(4): 591-617, 2012. [p380]

L. Anselin. Local indicators of spatial association-LISA. Geographical Analysis, 27(2):93-115, 1995. [p380]

M. P. Ashby. Studying Crime and Place with the Crime Open Database: Social and Behavioural Scienes. Research Data Journal for the Humanities and Social Sciences, 1(aop):1-16, 2019. [p386]

A. Baddeley, E. Rubak, and R. Turner. Spatial point patterns: methodology and applications with R. CRC Press, 2015. [p380, 381]

A. Baddeley, G. Nair, S. Rakshit, and G. McSwiggan. "Stationary" point processes are uncommon on linear networks. Stat, 6(1):68-78, 2017. [p380]

M. Bíl, R. Andrášik, and Z. Janoška. Identification of hazardous road locations of traffic accidents by means of kernel density estimation and cluster significance evaluation. Accident Analysis $\mathcal{E}$ Prevention, 55:265-273, 2013. [p383]

M. Bíl, R. Andrášik, T. Svoboda, and J. Sedoník. The KDE+ software: a tool for effective identification and ranking of animal-vehicle collision hotspots along networks. Landscape Ecology, 31(2):231-237, 2016. [p381]

R. Bivand and N. Lewin-Koh. maptools: Tools for Reading and Handling Spatial Objects, 2017. URL https: / / CRAN.R-project.org/package=maptools. R package version 0.9-2. [p383]

R. Bivand and C. Rundel. rgeos: Interface to Geometry Engine - Open Source ('GEOS'), 2020. URL https: //CRAN.R-project.org/package=rgeos. R package version 0.5-3. [p384]

R. S. Bivand, E. Pebesma, and V. Gómez-Rubio. Applied spatial data analysis with R, Second edition. Springer, NY, 2013. URL http: / /www . asdar-book.org/. [p380, 383]

Á. Briz-Redón. SpNetPrep: An R package using Shiny to facilitate spatial statistics on road networks. Research Ideas and Outcomes, 5:e33521, 2019. [p384, 386]

Á. Briz-Redón, F. Martínez-Ruiz, and F. Montes. Identification of differential risk hotspots for collision and vehicle type in a directed linear network. Accident Analysis E Prevention, 132:105278, 2019a. [p381, 383, 391]

Á. Briz-Redón, F. Martínez-Ruiz, and F. Montes. Spatial analysis of traffic accidents near and between road intersections in a directed linear network. Accident Analysis E Prevention, 132:105252, 2019b. [p380]

S. Chainey, L. Tompson, and S. Uhlig. The utility of hotspot mapping for predicting spatial patterns of crime. Security Journal, 21(1-2):4-28, 2008. [p382]

M. Deng, X. Yang, Y. Shi, J. Gong, Y. Liu, and H. Liu. A density-based approach for detecting networkconstrained clusters in spatial point events. International Journal of Geographical Information Science, 33(3):466-488, 2019. [p381]

P. J. Diggle. Statistical Analysis of Spatial and Spatio-Temporal Point Patterns. CRC press, 2013. [p380]

D. H. Douglas and T. K. Peucker. Algorithms for the reduction of the number of points required to represent a digitized line or its caricature. Cartographica: the International Journal for Geographic Information and Geovisualization, 10(2):112-122, 1973. [p384]

G. Drawve and A. Wooditch. A research note on the methodological and theoretical considerations for assessing crime forecasting accuracy with the predictive accuracy index. Journal of Criminal Justice, page 101625, 2019. [p383]

M. Eckardt and J. Mateu. Point patterns occurring on complex structures in space and space-time: An alternative network approach. Journal of Computational and Graphical Statistics, 27(2):312-322, 2018. [p381] 
M. Eckardt and J. Mateu. Second-order and local characteristics of network intensity functions. TEST, 30(2):318-340, 2021. [p381]

A. Getis and J. Ord. The Analysis of Spatial Association by Use of Distance Statistics. Geographical Analysis, 24(3), 1992. [p380]

G. Grolemund and H. Wickham. Dates and Times Made Easy with lubridate. Journal of Statistical Software, 40(3):1-25, 2011. URL http://www. jstatsoft.org/v40/i03/. [p387]

V. Gómez-Rubio, J. Ferrándiz-Ferragud, and A. López-Quílez. Detecting clusters of disease with R. Journal of Geographical Systems, 7(2):189-206, 2005. [p380]

V. Gómez-Rubio, P. Moraga, J. Molitor, and B. Rowlingson. Dclusterm: Model-based detection of disease clusters. Journal of Statistical Software, Articles, 90(14):1-26, 2019. ISSN 1548-7660. doi: 10.18637/jss.v090.i14. URL https: / /www. jstatsoft.org/v090/i14. [p380]

R. J. Hijmans. raster: Geographic Data Analysis and Modeling, 2019. URL https : / / CRAN . R-project. org/package=raster. R package version 2.8-19. [p385]

J. E. Kelsall and P. J. Diggle. Kernel estimation of relative risk. Bernoulli, 1(1-2):3-16, 1995a. [p382]

J. E. Kelsall and P. J. Diggle. Non-parametric estimation of spatial variation in relative risk. Statistics in Medicine, 14(21-22):2335-2342, 1995b. [p382]

J. E. Kelsall and P. J. Diggle. Spatial variation in risk of disease: a nonparametric binary regression approach. Journal of the Royal Statistical Society: Series C (Applied Statistics), 47(4):559-573, 1998. [p381]

M. Kulldorff. A spatial scan statistic. Communications in Statistics-Theory and Methods, 26(6):1481-1496, 1997. [p380]

C. Lloyd. Spatial data analysis: an introduction for GIS users. Oxford University Press, 2010. [p382]

G. McSwiggan, A. Baddeley, and G. Nair. Kernel density estimation on a linear network. Scandinavian Journal of Statistics, 44(2):324-345, 2017. [p380, 381]

G. McSwiggan, A. Baddeley, and G. Nair. Estimation of relative risk for events on a linear network. Statistics and Computing, pages 1-16, 2019. [p381, 382]

S. Meyer, L. Held, and M. Höhle. Spatio-Temporal Analysis of Epidemic Phenomena Using the R Package surveillance. Journal of Statistical Software, 77(11), 2017. [p380]

M. M. Moradi, F. J. Rodríguez-Cortés, and J. Mateu. On kernel-based intensity estimation of spatial point patterns on linear networks. Journal of Computational and Graphical Statistics, 27(2):302-311, 2018. [p381]

M. M. Moradi, O. Cronie, E. Rubak, R. Lachieze-Rey, J. Mateu, and A. Baddeley. Resample-smoothing of Voronoi intensity estimators. Statistics and Computing, 29(5):995-1010, 2019. [p381]

K. Nie, Z. Wang, Q. Du, F. Ren, and Q. Tian. A network-constrained integrated method for detecting spatial cluster and risk location of traffic crash: A case study from Wuhan, China. Sustainability, 7 (3):2662-2677, 2015. [p380]

E. Pebesma. Simple Features for R: Standardized Support for Spatial Vector Data. The R Journal, 2018. URL https://journal.r-project.org/archive/2018/RJ-2018-009/index. html. [p383]

E. J. Pebesma and R. S. Bivand. Classes and methods for spatial data in R. R News, 5(2):9-13, November 2005. URL https://CRAN.R-project.org/doc/Rnews/. [p383]

S. Rakshit, A. Baddeley, and G. Nair. Efficient Code for Second Order Analysis of Events on a Linear Network. Journal of Statistical Software, 90(1):1-37, 2019a. [p381]

S. Rakshit, T. Davies, M. M. Moradi, G. McSwiggan, G. Nair, J. Mateu, and A. Baddeley. Fast Kernel Smoothing of Point Patterns on a Large Network using Two-dimensional Convolution. International Statistical Review, 2019b. [p381]

C. Schnell, A. A. Braga, and E. L. Piza. The influence of community areas, neighborhood clusters, and street segments on the spatial variability of violent crime in Chicago. Journal of Quantitative Criminology, 33(3):469-496, 2017. [p380] 
W. Steenbeek and D. Weisburd. Where the action is in crime? An examination of variability of crime across different spatial units in The Hague, 2001-2009. Journal of Quantitative Criminology, 32(3): 449-469, 2016. [p380]

L. van der Meer, L. Abad, A. Gilardi, and R. Lovelace. sfnetworks: Tidy Geospatial Networks, 2021. URL https: //CRAN.R-project.org/package=s fnetworks. R package version 0.5.2. [p383]

K. Walker. tigris: An R package to access and work with geographic data from the US Census Bureau. The R Journal, 8(2):231-242, 2016. [p384]

D. Weisburd. The law of crime concentration and the criminology of place. Criminology, 53(2):133-157, 2015. [p380]

Z. Xie and J. Yan. Kernel density estimation of traffic accidents in a network space. Computers, Environment and Urban Systems, 32(5):396-406, 2008. [p382]

Z. Xie and J. Yan. Detecting traffic accident clusters with network kernel density estimation and local spatial statistics: an integrated approach. Journal of Transport Geography, 31:64-71, 2013. [p380]

\author{
Álvaro Briz-Redón \\ Statistics and Operations Research \\ University of València \\ Spain \\ alvaro.brizeuv.es \\ Francisco Martínez-Ruiz \\ Statistics Office \\ City Council of València \\ Spain \\ fmartinezrevalencia.es \\ Francisco Montes \\ Statistics and Operations Research \\ University of València \\ Spain \\ francisco.monteseuv.es
}

